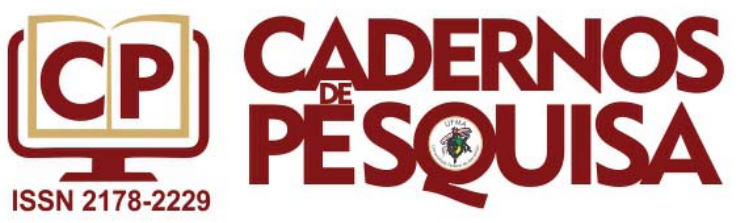

\title{
DESAFÍOS EPISTEMOLÓGICOS DE LA EDUCACIÓN SUPERIOR EN EL SIGLO XXI ${ }^{1}$ EPISTEMOLOGICAL CHALLENGES OF HIGHER EDUCATION IN THE 21ST CENTURY DESAFIOS EPISTEMOLÓGICOS DA EDUCAÇÃO SUPERIOR NO SÉCULO XXI
}

\author{
Alberto Matías González \\ Profesor Titular de la Universidad de Sancti Spíritus, José Martí Pérez, de Cuba. \\ albertomg1122@gmail.com \\ Orlando Fernández Aquino \\ Profesor-investigador del Programa de Pos-Graduación en Educación (Maestría \\ y Doctorado) de la Universidad de Uberaba (UNIUBE), MG. \\ Uberaba-MG, Brasil. \\ ofaquino@gmail.com
}

Resumen: Acudir a la epistemología es una práctica necesaria para el desempeño de la Educación Superior, más si se trata de la universidad en una sociedad cambiante, con interrogantes que echan por tierra las creencias con las que se han diseñado los sistemas educativos. El objetivo ha sido analizar la mudanza paradigmática que está ocurriendo en la actualidad en las ciencias, y en particular en la concepción del papel social de la Universidad, marcada por el surgimiento de epistemologías emergentes como el Pensamiento Complejo, el Movimiento Ciencia, Tecnología y Sociedad, la Epistemología del Sur y la Epistemología de Segundo Orden. El método ha sido el análisis hermenéutico de las fuentes consultadas. El resultado ha sido una visión sintética de la trasformación epistemológica en curso contenida en tendencias de pensamiento que, a pesar de sus diferencias, presentan coincidencias que muestran una ruptura con la epistemología positivista tradicional, la cual ha sido el sostén de formas de educación que han quedado obsoletas.

Palabras claves: Positivismo. Educación Superior. Epistemología.

Abstract: Return to the epistemology is a necessary practice for the performance of Higher Education, still more so in the case of the university in the mutant society with question that play by land the beliefs considered in the design of the educative systems. The objective of this study was to analyze the paradigmatic change that is happening currently in the sciences, and in particular in the conception of the social role of the University, marked by the emergence of emerging epistemologies such as Complex Thought, the Movement Science, Technology and Society, the Epistemology of the South and the Epistemology of the Second Order. The method was the hermeneutic analysis of the consulted sources. The result was a synthetic vision of the still in progress epistemological transformation expressed in trend of thought tendencies that, despite their differences, present coincidences that show a rupture with the traditional positivist epistemology, which has been the support of forms of education that are obsolete.

Keywords: Positivism. Higher education. Epistemology.

Resumo: Retomar a epistemologia é uma prática necessária para o desempenho da Educação Superior, mais ainda em se tratando da universidade numa sociedade mutante com interrogações que jogam por terra as crenças consideradas no desenho dos sistemas educativos. O objetivo deste estudo foi analisar a mudança paradigmática que está acontecendo na atualidade nas ciências e, particularmente, na concepção do papel social da universidade, marcada pelo aparecimento de epistemologias emergentes como o Pensamento Complexo, o Movimento Ciência, Tecnologia e Sociedade, a Epistemologia do Sul e a Epistemologia de Segunda Ordem. O método foi a análise hermenêutica das fontes consultadas. $O$ resultado foi uma visão sintética da transformação epistemológica em curso expressa em tendências de pensamento que, apesar de suas diferenças, apresentam coincidências que mostram uma ruptura com a epistemologia positivista tradicional, a qual tem sido a base de formas de educação que estão obsoletas.

Palavras-chaves: Positivismo. Educaçao Superior. Epistemologia.

1 Trabajo realizado con apoyo del Programa Nacional de Posdoctorado (PNPD) de la CAPES-Brasil.

Artigo recebido em junho 2017. Aprovado em novembro de 2017. 


\section{INTRODUCCIÓN}

Nunca antes todo lo concerniente al terreno de la Educación Superior ha sido tan debatido, se polemiza con todo: la organización de la universidad, la formación de profesores, las estrategias de enseñanza-aprendizaje, los modelos pedagógicos, la elaboración de proyectos y dentro de ello los fundamentos epistemológicos que sustentan el trabajo universitario.

La educación superior siempre se ha basado en cosmovisiones que incorporan teorizaciones con sus sistemas de preguntas y respuestas para legitimar las características de los programas de estudio, justificar los modelos y procedimientos didácticos y generar argumentaciones acerca de los fines de la universidad y el tipo de profesional que se pretende formar.

Al indagar en los orígenes de estas teorizaciones epistemológicas, encontramos una gran diversidad de influencias que, de manera abierta unas veces y ocultas en otras, obedecen a construcciones ideológicas que expresan los intereses de quienes concentran el ejercicio de mayores cuotas del poder.

A partir de los cambios operados en las últimas décadas asociados al desarrollo de las tecnologías, a la fragilidad de las profesiones tradicionales, al deterioro de principios éticos considerados universales, a las cambiantes y crecientes demandas de grupos y formaciones sociales, se introducen nuevas nociones que experimentan un cambio de sensibilidad y de configuración epistemológica en la Educación Superior. En esa perspectiva, el objetivo del presente trabajo es analizar la mudanza paradigmática que está ocurriendo -desde la segunda mitad del siglo XX hasta nuestros días-, en las ciencias y en particular en la concepción del papel social de la Universidad, marcada por tendencias de transición del pensamiento positivista para epistemologías emergentes como el Pensamiento Complejo, el Movimiento Ciencia Tecnología y Sociedad, la Epistemología del Sur y la Epistemología de Segundo Orden. Analizar cómo estas corrientes implican un cambio en el desempeño de las universidades es parte de los objetivos más específicos que los autores persiguen.

Como metodología se utilizó la investigación documental que incorporó una amplia revisión bibliográfica, cuyo resultado permitió la construcción de una síntesis teórica, que desde la crítica al positivismo recoge como las tendencias emergentes significan un cambio de paradigma en los conceptos de organización y funcionamiento de las universidades.

Reflexionar sobre las estructuras epistemológicas que sustentan el funcionamiento de las universidades es una actividad imprescindible, pues estas constituyen herramientas que facilitan procesos de organización, al mismo tiempo que hacen posible diseñar las características de los programas de las materias de aprendizaje, así como el concepto de egresado a que se aspira.

\section{EL POSITIVISMO COMO SOSTÉN EPISTEMOLÓGICO E IDEOLÓGICO DE LA UNIVERSIDAD TRADICIONAL}

El positivismo surge con Augusto Comte (1798-1857), en la tercera década del siglo XIX y se asocia a la ciencia experimental nacida en la modernidad capitalista, con el ofrecimiento de superar toda forma especulativa o metafísica en la ciencia. Esta exigencia debía ser extendida al entendimiento de la sociedad. El positivismo, asumido en el siglo XX bajo el rótulo de neopositivismo o empirismo lógico, ha jugado un rol importante en la configuración del discurso científico y de la educación en los últimos 150 años.

Desde finales del siglo XIX y a lo largo del XX, las sociedades más avanzadas de Europa y de América desarrollaron un espíritu modernizador, sustentado en el enfoque positivista. La noción de progreso asumida por esa visión contiene el optimismo triunfalista que, en ese período, sirvió para ocultar ingredientes tenebrosos. 
El positivismo, con su espíritu de objetividad y su reclamo por la sistematicidad y el rigor en el uso del método científico, promovió un enfrentamiento manifiesto contra la metafísica -concebida esta como razonamiento especulativo-, práctica tradicional que viene del pensamiento pre moderno, del cual la ciencia positivista debe apartarse. En este mismo orden de cosas sentimientos como el odio, el amor, la cobardía, la alegría y la tristeza pasan a ser desacreditados por su invalidez para aportar conocimiento verdadero y deben mantenerse alejados de la actividad de los científicos (MATÍAS; HERNÁNDEZ, 2014). Al decir de Alfred Ayer (1965, p. 126), "Los juicios de valor [...] en la medida en que no son científicos, no son, literalmente hablando, significativos, sino que son, simples expresiones de emoción que no pueden ser verdaderas ni falsas". De esta manera la subjetividad humana y en especial su capacidad valorativa quedaron declaradas como áreas de inutilidad para la ciencia.

Bajo la salvaguardia epistémica del positivismo, la ciencia moderna desarrolló los métodos empíricos. Se valió de las matemáticas y perfeccionó los sistemas estadísticos, útiles para hacer diagnósticos, evaluar resultados, identificar tendencias e incrementar la fiabilidad de los resultados. El fin consistía en lograr la objetividad en la producción científica, a partir del empleo acertado de las reglas del método científico, tomando como presupuesto esencial que objeto del conocimiento existe como una figura externa al sujeto. En este enfoque, el sujeto no interfiere en los resultados de la investigación, pues su papel debe limitarse a garantizar el empleo correcto del método científico.

Con estas herramientas que convocan al rigor, a la objetividad, a la fiabilidad, a la certeza, a la matematización y a la sobrevaloración de lo cuantitativo como aval, el positivismo asume una narrativa de neutralidad científica, que descarta la inclusión de la subjetividad en la investigación científica. A esto, se le une la intencionalidad de transferir los métodos de las ciencias naturales al análisis de la sociedad, justamente al campo donde la subjetividad es un contenido esencial de la existencia.

A partir esta noción racionalista que se configuró a través de los últimos siglos, se elaboró una imagen de la ciencia y del conocimiento científico que privilegia los valores cognitivos. El positivismo incorpora, también, a esta configuración argumental una comprensión lineal de la relación causa efecto, según la cual al conocer las causas de los fenómenos el hombre puede construir alternativas para influir o evitar los efectos, según considere (MATÍAS; HERNÁNDEZ, 2014). En realidad esta imagen, que hunde sus raíces en la tradición de los pensadores clásicos griegos, ha estado en la base de muchos de los resultados científicos y tecnológicos de la era moderna; sin embargo, también ha sido la causa de muchos fracasos, pues los resultados pueden ser inesperados y los esfuerzos investigativos pueden causar males no esperados.

Aunque la generalidad de los autores positivistas asumieron un discurso relativamente progresista a través de la práctica del antifascismo y en defensa de la paz, su filosofía a la larga ha establecido estrechas conexiones con las políticas neoliberales, siendo esta su expresión política más extrema, al configurarse en estructuras tecnocráticas que van tramando sutiles formas de dominación hegemónica, relacionadas al capitalismo corporativo y transnacional. Como resultado, una concepción y una práctica interiormente reaccionaria orientada a desmantelar el pensamiento crítico y cultivar el conformismo, la pasividad y la desmovilización social (MATÍAS; HERNÁNDEZ, 2014).

Como propuesta epistemológica, el positivismo ha mantenido una centralidad entre aquellos sectores sociales vinculados a la profesión científica y a la educación, centralidad que llega hasta nuestros días. Sin embargo, como enfoque de pensamiento filosófico su significado ha venido menguando desde la década de 1960. Varios acontecimientos han incidido en este declive, en especial el impacto de la obra de Tomas Kuhn La estructura de las revoluciones científicas, además de la interpretación de los desastres tecnológicos y sus impactos medioambientales y el surgimiento de los nuevos movimientos sociales que amplían el arco iris de las demandas por la libertad, para lo cual se precisan nuevas nociones explicativas. 


\section{INFLUENCIA DE LA EPISTEMOLOGÍA POSITIVISTA EN LA EDUCACIÓN SUPERIOR}

A partir de la década de 1980, con el advenimiento del neoliberalismo, el racionalismo instrumentalista se ensambló en el discurso y en la práctica política de Europa, Estados Unidos y de la mayoría de los países latinoamericanos. El neoliberalismo, apoyándose en el racionalismo positivista colocó el mercado como centro de sus preocupaciones, causando terribles males para los sectores sociales desfavorecidos en diferentes partes del mundo.

Esta fusión entre epistemología positivista y políticas neoliberales es el resultado de una evolución histórica. A lo largo del siglo XIX y hasta bien entrado el XX, se consolidó el modelo de universidad que llega hasta nuestros días y que propició este empalme en las Instituciones de Educación Superior (IES). Los antecedentes más remotos de este proceso lo tuvieron las universidades alemanas, configuradas a partir de la noción de ciencia pura, consagrada por profesores expertos y estudiantes que se preparaban para ejercer una profesión especializada, con título de expertos en una materia, a partir de currículos impuestos. La organización fue estructurada en facultades y departamentos, con enfoques de ciencia disciplinar.

Este modelo de universidad, unido a los procesos de especialización que han tenido lugar en la ciencia y la tecnológica a lo largo de los siglos XIX y XX, favoreció la parcelación del conocimiento. Un producto de estos procesos de fragmentación cognitiva fue la formación de dos grandes culturas, una humanística y otra de ciencias naturales y exactas, con escasos canales internos de comunicación y términos epistémicos que no pueden ser quebrantados. Esto fue descrito y condenado por Paul Snow (1964) a inicios de la década de 1960. La epistemología positivista desde el menosprecio a las humanidades vino así a respaldar esta organización, que aún es dominante en la mayoría de las universidades del mundo.

Cada uno de los campos académicos, se institucionalizó por medio de la creación de un ritual científico que incorporó conferencias, grupos de investigación con sus proyectos, eventos, publicaciones y sistemas de estímulo a la producción y difusión del conocimiento. Todo ello para mostrar los resultados alcanzados con dimensiones locales, nacionales e internacionales. A esta configuración se le asoció la elaboración de currículos académicos, para destacar el ascenso y la producción de los profesionales involucrados, y también muchas veces de los futuros graduados.

El modelo quedó diseñado con un profesor universitario productor y difusor de conocimientos verdaderos y universales y un alumno receptor de los mismos, así como de los discursos políticos dominantes. La docencia se organizó en disciplinas académicas inflexibles en las cuales los alumnos deben obedecer, pues los expertos son la verdadera y única autoridad.

Este tipo de universidad generalmente ha excluido cualquier otra forma de saber que salga de los marcos del conocimiento científico y tecnológico, generando procesos de homogenización del saber en los que se marginan las tradiciones culturales nacidas de la diversidad social e histórica.

El impacto de la fusión entre la epistemología positivista y el discurso político neoliberal tiene efectos muy diversos en el desempeño de la Educación Superior, de los cuales es muy difícil escapar. En este escenario la universidad se convierte en una pieza más de los mecanismos del mercado global y pasa a ser una empresa con capacidad competitiva que se ve obligada a modificar sutilmente sus tradicionales rituales en función de ascender posiciones en los listados del ranking internacional de universidades. Esto implica la institucionalización de estratégicas que abarcan diversas áreas en su funcionamiento.

Es precisamente la acreditación universitaria, como instrumento evaluativo, el principal mecanismo utilizado para compulsar las metas de mejoramiento y competitividad exigidas, proceso que incorpora toda una serie indicadores que deben ser contabilizaos: 
calidad de los procesos de formación de profesionales, existencia de proyectos de investigación, cantidad profesores con título de doctores y master, publicaciones en revistas indexadas y de alto impacto, captación de ingresos económicos e infraestructura lograda.

En cuanto a la calidad de los procesos de formación de profesionales, la universidad neoliberal impone una noción de calidad que involucra aspectos de los sistemas empresariales: mecanismos de control, mejoramiento y aseguramiento de la calidad, además de términos como clientes, partes interesadas, proveedores. Con todas estas denominaciones tecnocráticas se insinúa sutilmente que la educación es una empresa, que produce mercancías que pueden ser acreditadas por agencias comerciales.

Para ello, el mestizaje natural que se produce entre el espíritu positivista que preferencia los valores cognitivos del conocimiento y la lógica mercantil del neoliberalismo, reafirma un elitismo en el funcionamiento de la Educación Superior que no solo patrocina un aprendizaje despojado de intereses políticos o éticos, sino que defiende también que a los estudiantes no se les debe enseñar teoría en demasía, ni habilidades de pensamiento crítico, por lo que la historia, la literatura y la filosofía como materias no deben aparecer en los currículos. Con ello, formar se reduce a crear un "producto" económico para el mercado de trabajo, que prescinde del sujeto autoreflexivo, cuyo desempeño viene acompañado del dogma según el cual este mundo es el mejor de todos los posibles.

Esta lógica se complejiza en el ejercicio de producción de conocimientos, pues sirve de base a la organización de proyectos financiados por el Estado, ONGs y empresas privadas. Las aprobaciones de dichos proyectos generalmente privilegian a las instituciones con más capacidad para obtener resultados y dentro de ellas las áreas de las ciencias naturales y de las ingenierías. Es una lógica que en los países de la periferia termina reproduciendo estructuras coloniales, pues son los grandes centros culturales los que más aportan conocimiento de interés, con cargas políticas intencionadas que las periferias terminan asimilando.

En cuanto a las publicaciones que salen de las investigaciones que realizan los profesores y los alumnos que participan en los proyectos, indicador este fundamental para los procesos de acreditación y ubicación en el ranking universitario internacional y nacional, el mercantilismo y la banalización se convierten en una práctica que consolida el elitismo y ronda con la hipocresía, hasta el punto de llegar a considerar que un paper redactado tal vez en dos o tres meses y publicado en una revista de alto impacto tiene mayor valor que un libro que puede haber sido el resultado de una investigación de diez años. Esto condiciona en los docentes investigadores un mayor interés por las revistas indexadas, al mismo tiempo que el desinterés por las revistas locales, lo cual puede acontecer independientemente de la calidad real que tengan los trabajos. Igual sucede con las citaciones, una citación en una revista bien ubicada en los índices de Scopus o en la Web of Science se le atribuye mayor significado que una que no lo esté.

Otra trascendencia del impacto de la asociación del positivismo y el neoliberalismo lo encontramos en los procesos de fusión de universidades. Varios países (Estonia, Chile, Finlandia, Dinamarca, Bélgica, Suecia, Francia, por citar algunos) desarrollan procesos de unificación de universidades, con el fin de concentrar los talentos y recursos, tener más visibilidad y ascender en el ranking.

Esta mercantilización ha sido objeto de muchas críticas, (BOURDIEU, 1999; LANDER, 2008) por considerar que refuerzan los paradigmas epistemológicos coloniales dominantes. Sin embargo, el conjunto de creencias que la legitiman se han ido normalizando y aceptando como lo natural. 


\section{LOS CAMBIOS DEL ESCENARIO SOCIAL EN QUE EXISTE LA UNIVERSIDAD ACTUAL}

La realidad epistemológica descrita, dominada por el racionalismo instrumentalista, el mercado y la privatización, instalada en las últimas décadas del siglo XX en la educación superior, no da respuesta a las realidades cambiantes ni a los desafíos que plantea el siglo XXI.

El proceso de planetarización de la economía y la cultura, así como los reclamos de cada contexto, exigen modificaciones sensibles al funcionamiento, la misión y los fines de la universidad.

Es muy visible el poder alcanzado por el desarrollo científico y tecnológico, que está transformando la vida de los seres humanos con una doble marca: de un lado la realización de potencialidades que mejoran considerablemente la vida de los humanos; de otro lado, y sin que pueda separarse de lo anterior, se desarrollan capacidades destructivas e inseguras asociadas a la energía nuclear y la biotecnología.

La informatización invade de manera desigual los diversos campos y espacios de la actividad humana, el volumen de información disponible se ha multiplicado con creces, la comunicación en tiempo real ha borrado las distancias, las cámaras invaden los espacios sociales favoreciendo mecanismos de control y vigilancia nunca antes pensados, los conceptos de amistad y el contacto personal se modifican con las redes sociales, pues ahora puede ser por cable, favoreciendo la tendencia a la renuncia al discurso hablado, a la construcción del mundo a través de la palabra y asumirnos como seres audiovisuales. Asociado a este nuevo mundo tecnológico crece la privatización del conocimiento a través de los mecanismos de propiedad intelectual, que estimulan al mundo empresarial a invertir en la producción de conocimiento, por lo que la universidad pierde la posición privilegiada de que había gozado desde su surgimiento en la investigación, pero al mismo tiempo su función cómo productora de conocimiento crece pues la calidad de la formación depende de ello.

En el mundo laboral la fugacidad, el desvanecimiento o como lo llama Zygmunt Bauman (2004) modernidad líquida, aparece como una coordenada de desestabilización de las profesiones y obliga a cambiar las estrategias de vida de los individuos para poder adaptarse a las nuevas reglas y al mismo tiempo obliga a las universidades a modificar sensiblemente su desempeño. Al respecto Carlos Delgado (2009, p. 1) señala que:

[...] el egresado en estos momentos se enfrenta a que su preparación no coincide algunas veces con las exigencias de su perfil laboral, y por tanto, si no se le brinda al alumno un tipo de flexibilidad que le permita reincorporarse al proceso productivo, debe empezar de nuevo [...] los plazos entre la introducción de un avance y otro se acortan $[\ldots]$ entonces debo estar preparado para ese otro avance.

Nos encontramos, en fin, con una sociedad que produce, vende y consume mediocridad y superficialidad de todo tipo: mercantilización de casi todo, obsesión por el consumo de objetos de marcas reconocidas, sustitución de los héroes fundadores de nuestras naciones o de la ciencia por los famosos del fútbol o del modelaje, cultura del sexo que subordina la vida al cuerpo y lo erotiza. Se trata de una realidad fantasiosa y de apropiación acrítica del medio cultural, cuya realización nos lleva a una parálisis intelectual que produce escenarios idóneos para la recolonización cultural.

¿Cómo se puede atender, con la seriedad requerida, esta compleja realidad desde la universidad? Es una interrogante que evidencia la crisis de un paradigma epistemológico que aún sigue siendo dominante, al mismo tiempo que la necesidad de nuevos argumentos epistemológicos que fundamenten la razón de ser de la Educación Superior actual. 


\section{EL GIRO EPISTEMOLÓGICO DE LAS ÚLTIMAS DÉCADAS}

La realidad epistemológica de la educación superior del siglo XXI necesita refundar las bases que la legitiman, por lo que reinterpretar la sociedad y el papel de la universidad es una tarea de indudable actualidad. A pesar de que el ideal positivista en el mundo universitario sigue siendo dominante, lo nuevo se hace cada vez más perceptible, su espíritu es apreciable en diversas narrativas. Cuatro líneas de ruptura permiten constatar el cambio hacia un Nuevo Saber. Ellas son:

a) la formulación de problemas de nuevo tipo en los límites del conocimiento científico y en la vida social;

b) el acercamiento mutuo del conocimiento científico social y natural en los nuevos cuestionamientos teóricos sobre los límites de la ciencia occidental;

c) el replanteo del objeto de la ciencia como asunto metodológico y ético;

d) las soluciones teóricas innovadoras de la Bioética Global, el Holismo Ambientalista, la Nueva Epistemología y el enfoque de la Complejidad (SOTOLONGO; DELGADO, 2006, p. 31).

Las evidencias de nuevas estructuras epistemológicas también se aprecian en el discurso político del Buen Vivir de líderes como Evo Morales y Rafael Correa, en la propuesta y práctica del discurso de la Agroecológica y de la Permacultura por agricultores y académicos, en la narrativa Decolonial de Boaventura de Sousa, Enrique Dussel, Santiago Castro-Gómez y Ramón Grosfoguel y en el movimiento Ciencia, Tecnología y Sociedad (CTS).

Este giro epistemológico contiene diferencias que son el resultado de una realidad social diversa, caracterizada por la presencia de inquietudes e interrogantes emergentes que expresan antagonismos inéditos junto otros que vienen de épocas anteriores, así como por el surgimiento de nuevos movimientos sociales: ecologistas, indigenistas, femeninitas, pacifistas, por los derechos de orientación sexual (LGTB), contra la discriminación racial, campesinos, anticorrupción, etc. Sin embargo, como se podrá apreciar contienen elementos comunes que permiten identificarlos como figuras epistemológicas alternativas a la tradición positivista neoliberal.

Su impacto en la Educación Superior ha sido creciente, pues las terminologías y argumentos que conforman las estructuras epistemológicas de lo que se ha dado en Ilamar Revolución Contemporánea del Saber (SOTOLONGO; DELGADO, 2006), penetran las filosofías que sustentan las políticas que sirven de guía en el desempeño de las universidades y por otra parte muchos de los representantes de las nuevas concepciones son profesores e investigadores universitarios que organizan sus conferencias, congresos, publicaciones e introducen las ideas que defienden en los programas que imparten. A continuación hacemos referencia a algunas de las líneas de desarrollo teórico que más están impactando la Educación Superior.

Los estudios Ciencia, Tecnología y Sociedad (CTS). En la década de 1960, bajo la influencia de las amenazas provenientes de la ciencia y la tecnología, surgen los CTS como campo de trabajo en la investigación académica, educativa y de políticas públicas. Se trata de un enfoque interdisciplinar que promueve:

- La democratización de la gestión, del control, la toma de decisiones y la difusión del conocimiento científico y tecnológico, a fin de socavar las formas monopólicas en el uso del conocimiento, hacer frente a las amenazas que provienen de la ciencia y la tecnología y acabar con el elitismo en el aprendizaje a través de procesos de alfabetización en ciencia y tecnología (GONZÁLEZ; LÓPEZ; LUJÁN, 1997).

- El reconocimiento de las dimensiones sociales en la ciencia y la tecnología, a fin de superar visiones reduccionistas (AIBAR; QUINTANILLA, 2002; GONZÁLEZ; LÓPEZ; LUJÁN, 1997). 
- La contextualización del conocimiento, a partir del argumento que considera que la pertinencia de la ciencia y la tecnología nace de su correspondencia con las tradiciones y la cultura de los pueblos y de que la universidad debe integrarse a la solución de las necesidades locales.

- La evaluación del condicionamiento y el impacto social de los desarrollos de la ciencia y la tecnología (GONZÁLEZ; LÓPEZ; LUJÁN, 1997).

- Un enfoque interdisciplinar que integre ciencias humanísticas y naturales y exactas y valorice perspectivas históricas de análisis en el desarrollo de la ciencia y la tecnología.

- La sensibilidad en relación con los problemas de contaminación ambiental, a fin de generar una conciencia global de un problemática que es global.

- El despertar de una percepción de riesgo ante el desarrollo de la ciencia y la tecnología, al considerar que es un desarrollo acompañado de incertidumbres.

- Una percepción en valores en la comprensión de ciencia y tecnología que incluyen los valores éticos, políticos, económicos, militares, etc., del conocimiento. La ciencia es una actividad transformadora del mundo, que no se limita a la indagación de cómo es el mundo, sino que trata de modificarlo en función de valores y fines (ECHEVERRÍA, 1995, p. 68).

Los CTS se han ido consolidando institucionalmente en la Educación Superior de muchos países, a través de la promoción del campo de estudios CTS con la creación de programas de maestrías y doctorados y de la incorporación de los estudiantes a la búsqueda de soluciones, el desarrollo de programas de investigación en dicho campo, la incorporación de materias humanísticas en las carreras de ingeniería, el establecimiento de currículos más flexibles que incorporan los intereses de los estudiantes, quienes pueden llegar a ser coautores de sus planes de estudio y otras variantes a veces muy particulares de las universidades.

Una de las ventajas del enfoque CTS en la universidad es el compromiso que establece con el estímulo al pensamiento crítico, referido a la compresión del desarrollo científico y tecnológico. Con ello se pretende promover el ejercicio ciudadano, comprometido y responsable en la evaluación y toma de decisiones en ciencia y tecnología, además de valorar sus impactos en la vida de las personas y en el medio natural donde se desarrolla.

El pensamiento complejo. La noción de pensamiento complejo fue introducida por el filósofo francés Edgar Morin (1921), desde finales de la década de 1960, convirtiéndose en una visión de moda en los 90. Ilya Prigogine (1917-2003) y Humberto Maturana (1928) también han aportado argumentos de valor a la teoría de la complejidad. La complejidad es expresión de la crisis que vive la sociedad contemporánea, aunque tiene puntos de contacto con la dialéctica materialista de Carlos Marx. Abarca el análisis de una serie de preocupaciones que desbordan la cuestión social de la lucha de clases, el desarrollo o la cuestión nacional, aspectos centrales en el pensamiento marxista.

Características de la noción de pensamiento complejo:

- Reconsidera las nociones de determinismo, causalidad y pronóstico, desde la crítica a la visión lineal en la interpretación de la relación causa efecto.

- Comprende el universo como una red de relaciones, interdependientes, multidimensionales e interactivas, ante las cuales el conocimiento humano siempre será impreciso, al mismo tiempo que escenario de incertidumbres al lado de certidumbres "El conocimiento, sigue siendo la navegación en un océano de incertidumbres a través de un archipiélago de certezas". (MORIN, 1999, p. 50).

- Promueve un enfoque transdisciplinario y holístico. 
- Hace uso de la cibernética, la teoría del caos, el enfoque de sistemas, la noción de dinámica no lineal y la epistemología de segundo orden.

- Promueve el diálogo de saberes, entre culturas, entre las disciplinas científicas, entre las mal llamadas ciencias duras y ciencias blandas, entre el saber científico y el no científico.

- Postula principios fundamentales como la dialogía, que se refiere al vínculo entre sistemas; la recursividad, que expresa la capacidad de modificación de los sistemas como resultado de interacciones diversas y; la hologramía, que expresa la conexión del todo y las partes.

- Promociona el humanismo sobre la base de la necesidad de fundar una ética planetaria.

- Estimula la formación y desarrollo del pensamiento crítico y la cultura reflexiva, como fundamento para la construcción de una sociedad capaz de buscar las mejores soluciones a la crisis actual.

Esta noción considera que la universidad debe romper definitivamente con la imagen de un mundo parcelado, a través de un proceso de tránsito permanente hacia formas de integración del saber, lo que presume incorporar enfoques de complementariedad, intercepción y aproximación del conocimiento. Esta consideración dirigida al ejercicio de las universidad plantea cuestionamientos y políticas de ruptura ante las tradicionales estructuras de organización universitaria en facultades, departamentos y disciplinas; asumiendo propuestas más flexibles que incorporan a un tronco común diversas disciplinas como los colectivos de carreras, los injertos de materias humanísticas en las ciencias técnicas y naturales, la idea de que el estudiante participe en la conformación de su propio currículo, las investigaciones temáticas que involucran las diversas dimensiones socio ambientales.

El enfoque de complementariedad y aproximación del conocimiento es expresión, en una dinámica de retroalimentación, del escenario social actual: deterioro de las relaciones hombre-naturaleza, que exigen soluciones de saber integrado; desarrollos tecnológicos que precisan de soluciones éticas y políticas novedosas, pues el especialista tradicional es incapaz de dárselas; fenómenos sociales como la violencia y las drogas, de complejidad tal que exigen la concurrencia de saberes diversos e integrados para encontrar soluciones realistas; espacios laborales que necesitan de profesionales más integrales, de pensamiento creativo y flexible, pues los escenarios donde se insertan son cambiantes.

En materia de educación, el pensamiento complejo también se proyecta por una profunda reforma, que por supuesto incluye al papel social de las universidades, en especial la revisión del papel y los ideales de los profesores: "la reforma educativa ha de fundirse con la reforma del pensamiento, de la política y lo político. En ello radica su reinvención" (MORIN; DELGADO, 2014, p. 17). Lo cual significa un enorme reto, pues implica deconstruir, desorganizar los mecanismos tradicionales de desempeño caracterizados por el aprendizaje repetitivo y crear una cultura educativa donde el maestro actúe como un guía creativo del alumno, quien a su vez participa de su propio autoaprendizaje.

El pensamiento complejo también adopta el concepto de multidiversidad, como alternativo al de universidad, que significa un espacio educativo abierto, flexible, dispuesto al diálogo en busca de perspectivas nuevas que sean capaces de religar el conocimiento y donde puedan coexistir saberes múltiples que hagan posible entender el mundo como una realidad de fenómenos entrelazados. Este empeño se acompaña de una reconstrucción de los paradigmas éticos sobre los que se levanta la Educación Superior.

La narrativa Decolonial o Epistemología del Sur. Las perspectivas teóricas de lo que se ha dado en llamar descolonial o decolonial, identificado también como Epistemología 
del Sur hacen alusión a un grupo importante de estructuras conceptuales nacidas en las últimas décadas. El movimiento surge como contrapartida a la modernidad colonial (MIGNOLO, 2008), e implica una construcción epistemológica que incorpora un proyecto político y un sujeto, que sin negar los valores del pensamiento occidental, desafían su espíritu eurocentrista colonizador.

\begin{abstract}
El giro de-colonial es la apertura y la libertad del pensamiento y de formas de vida (economías-otras, teorías políticas-otras), la limpieza de la colonialidad del ser y del saber; el desprendimiento del encantamiento de la retórica de la modernidad, de su imaginario imperial articulado en la retórica de la democracia. (MIGNOLO, 2008, p. 252).
\end{abstract}

El giro decolonial ampara la legitimidad de un diálogo de saberes, que incorpore las tradiciones de pensamiento y las formas de existencia de los pueblos del Sur y en especial de las comunidades de origen africano y aborigen, como forma de derrumbamiento de lo colonial en el conocimiento y las instituciones sociales y políticas.

En cuanto al discurso político, el pensamiento decolonial dirige su mirada hacia el concepto de emancipación social en época de transición, concepto que identifica como principal encargo de la universidad; pero reconoce que para que se pueda cumplir ese encargo se deben remover los fundamentos epistemológicos que han sostenido la educación en los últimos siglos.

\begin{abstract}
Nuestra situación es algo compleja: podemos afirmar que tenemos problemas modernos para los cuales no tenemos soluciones modernas. Y esto le da a nuestro tiempo el carácter de transición: tenemos que hacer un esfuerzo muy exigente por reinventar la emancipación social. (SOUSA, 2006, p. 15).
\end{abstract}

Es por ello, que categorías como libertad, emancipación, derechos, ciudadanía no pueden ser entendidas de manera estática, al mismo tiempo que la "educación no es solamente una condición de acceso a los puestos de trabajo o a las posiciones sociales, es la condición mayor de acceso al ejercicio verdadero de los derechos del ciudadano" (SOUSA, 2006, p. 48).

Con este discurso epistemológico se fomenta una ruptura radical con el logocentrismo característico de la tradición occidental que ha sido servido en las universidades, es una propuesta que se esfuerza por contextualizar el saber, al mismo tiempo que trata de mostrar la pertinencia actual del legado construido a través de la historia de pueblos que nunca han sido escuchados.

Por otra parte, la Epistemología del Sur está condicionando el surgimiento de universidades para atender las necesidades educativas de los pueblos aborígenes, las cuales basan su desempeño en preceptos como: la transdisciplinariedad, el enfoque intercultural y transcultural, el diálogo de saberes, la descolonización del conocimiento, traducción entre saberes, ecología de saberes, aprendizaje colectivo. Estos preceptos determinan en las nuevas universidades los currículos y el estilo de trabajo que en su lucha por el reconocimiento de la diferencia operan contra la discriminación racial, étnica o de clase.

Epistemología de segundo orden. Aunque los gérmenes de los ideales del pensamiento de segundo orden pueden ser encontrados en décadas anteriores, pero es a partir de 1970 con Heinz Von Foerster y la cibernética de segundo orden que comienza a hablarse de ello. Esta se basa en el presupuesto de la unidad del sujeto y el objeto como definibles exclusivamente en su relación, por lo que los resultados del proceso de conocimiento implican la dinámica de ambos elementos. El interés no solo está en lo observado sino que se incluye el observador en el sistema observado. Según Foerster (apud MORIN, 1999, p. 31) "no sólo una epistemología de los sistemas observados, sino también una epistemología de los sistemas observadores". En este enfoque el sujeto es parte de la misma realidad que investiga y su presencia puede alterar la observación. 
En resumen, sujeto y objeto no son separables, sino que coexisten y se interpenetran, ya que el sujeto y sus valores están implicados; el objeto es dinámico y reacciona a la acción del sujeto. Esto significa, en esencia, un cuestionamiento al enfoque tradicional de objetividad que el positivismo respalda, en que un sujeto apartado del objeto y aplicando correctamente el método científico produce conocimiento objetivo, sin las interferencias del sujeto.

La matriz epistemológica de segundo orden tiene un gran impacto en la Educación Superior, pues reconsidera las bases epistémicas más profundas de la producción de conocimiento, así como de su interpretación. El cambio de la noción de objetividad, tradicionalmente exigida por profesores y evaluadores en los resultados científicos y académicos, implica para el ejercicio docente e investigador de la universidad una profunda modificación. Por otra parte, incorpora como elementos esenciales la noción de incertidumbre, el espíritu de cooperación, así como la práctica de enfoques de integración, complementariedad y aproximación del conocimiento.

\section{CONCLUSIONES}

El paradigma epistemológico del positivismo que sirvió de fundamento al funcionamiento de la academia y de la Universidad en el mundo occidental, hasta la segunda mitad siglo XX, comienza ser estremecido por la emergencia de nuevas epistemologías como el Pensamiento Complejo, el Movimiento Ciencia Tecnología y Sociedad, la Epistemología del Sur y la Epistemología de Segundo Orden.

La emergencia de estas perspectivas de cambio epistemológico, independientemente de sus diferencias argumentativas y fines sociales, tienen en común: la reprobación del pensamiento occidental tradicional y su modelo de vida; la implementación de estrategias de integración del conocimiento; la comprensión del conocimiento y de la vida como consideraciones éticas, en función del nacimiento de una nueva subjetividad; la intencionalidad de desarrollar el pensamiento crítico, a partir de la noción de aprender y enseñar a pensar, así como de la necesidad de reinterpretar y buscar nuevas explicaciones para participar de la transformación social.

Para que la universidad responda a estas demandas debe transformarse como institución social y científica. Esto significa reconstruir la Educación Superior y su lugar en el entramado socioeconómico; así como redefinir su misión transsecular en la formación de nuevos profesionales, en la conservación, producción y diseminación de los nuevos conocimiento en sus diferentes modalidades.

Instrumentar el debate crítico en la formación de profesores sobre contenidos de epistemología, es tal vez la recomendación más pertinente para participar de la superación del legado positivista en la Educación Superior. Es a través de la discusión que la comunidad universitaria puede tomar conciencia de la importancia que tiene asumir paradigmas que incorporen dimensiones sociales en la comprensión del conocimiento

\section{AGRADECIMENTOS}

Ao Programa Nacional de Pós-doutoramento (PNPD), da CAPES, e à Fundação de Amparo à Pesquisa do Estado de Minas Gerais (FAPEMIG) pelo apoio recebido para realização desta pesquisa. 


\section{REFERENCIAS}

AIBAR, E.; QUINTANILLA, M. Á. Cultura tecnológica: estudios de ciencia, tecnología y sociedad. Barcelona, España: ICE Universidad de Barcelona; Horsori, 2002.

AYER, A. Lenguaje, verdad y lógica. Buenos Aires, Argentina: EUDEBA, Editorial Universitaria, 1965.

BAUMAN, Z. Modernidad líquida. México; Argentina: Fondo de Cultura Económica, 2004.

BOURDIEU, P. Dos imperialismos de lo universal. In: . Intelectuales, política y poder. Buenos Aires, Argentina: Eudeba, 1999.

DELGADO, C. J. El pensamiento de Marx es compatible con la teoría de la complejidad. Juventud Rebelde, Cuba, viernes 23 de enero de 2009. Entrevista concedida al periodista Giovanni Fernández Valdés.

ECHEVERRÍA, J. Filosofía de la ciencia. Madrid, España: Akal, 1995.

GONZÁLEZ, M.; LÓPEZ, J. A.; LUJÁN, J. L. Ciencia, tecnología y sociedad. Barcelona, España: Ariel, 1997.

LANDER, E. La ciencia neoliberal. Tabula Rasa, Bogotá, Colombia, n. 9, p. 247-283, 2008.

LUJÁN, J. L.; LÓPEZ, J. A. Ciencia y valores en la regulación del cambio tecnológico. In: AlBAR, E.; QUINTANILLA, M. A. Ciencia, tecnología y sociedad. España: Editorial Trotta, 2012. p. 277-302.

MATÍAS, A.; HERNÁNDEZ, A. Positivismo, dialéctica materialista y fenomenología: tres enfoques filosóficos del método científico y la investigación educativa. Actualidades Investigativas en Educación, Costa Rica, v. 14, n. 3, 2014. Disponible en: <http://revistas.ucr.ac.cr/index. php/aie/article/view/16155/18115>. Acceso en: 07 fev. 2017.

MIGNOLO, W. El pensamiento decolonial: desprendimiento y apertura: un manifiesto. Tabula Rasa, Bogotá, Colombia, n. 8, p. 243-281, 2008.

MORÍN, E.; DELGADO, C. Reinventar la educación: abriendo caminos a la metamorfosis de la humanidad. México: Multiversidad Mundo Real Edgar Morín, 2014.

MORIN, E. El Método III: el conocimiento del conocimiento. Madrid, España: Ediciones Cátedra, 1999.

SNOW, P. Las dos culturas y un segundo enfoque. Madrid, España: Alianza, 1964.

SOTOLONGO, P. L.; DELGADO, C. J. La revolución contemporánea del saber y la complejidad social. Buenos Aires, Argentina: CLACSO, 2006.

SOUSA, B. Renovar la teoría crítica y reinventar la emancipación social. Buenos Aires, Argentina: CLACSO, 2006. 